\title{
Functional outcomes of adults with 22q11.2 deletion syndrome
}

\author{
Nancy J. Butcher, MSC ${ }^{1,2}$, Eva W.C. Chow, MD, MPH ${ }^{1,3}$, Gregory Costain, MSc ${ }^{1,2}$, Dominique Karas, MHS1, \\ Andrew Ho ${ }^{1}$ and Anne S. Bassett, MD ${ }^{1-5}$
}

Purpose: The 22q11.2 deletion syndrome is a common multisystem genomic disorder with congenital and later-onset manifestations, including congenital heart disease, intellectual disability, and psychiatric illness, that may affect long-term functioning. There are limited data on adult functioning in 22q11.2 deletion syndrome.

Methods: We used the Vineland Adaptive Behavior Scales to assess functioning in 100 adults with 22q11.2 deletion syndrome ( $n=46$ male; mean age $=28.8$ (standard deviation $=9.7$ ) years) where intellect ranged from average to borderline $(n=57)$ to mild intellectual disability $(n=43)$.

Results: More than $75 \%$ of the subjects scored in the functional deficit range. Although personal, vocational, and financial demographics confirmed widespread functional impairment, daily living skills and employment were relative strengths. Intelligence quotient was a significant predictor $(P<0.001)$ of overall and domain- specific adaptive functioning skills. A diagnosis of schizophrenia was a significant predictor $(P<0.05)$ of overall adaptive functioning, daily living skills, and socialization scores. Notably, congenital heart disease, history of mood/anxiety disorders, sex, and age were not significant predictors of functioning.

Conclusion: Despite functional impairment in adulthood that is primarily mediated by cognitive and psychiatric phenotypes, relative strengths in activities of daily living and employment have important implications for services and long-term planning. These results may help to inform expectations about outcomes for patients with 22q11.2 deletion syndrome.

Genet Med 2012:14(10):836-843

Key Words: 22q11 deletion syndrome; adaptive functioning; heart defects; congenital; intellectual disability; schizophrenia

\section{INTRODUCTION}

The 22q11.2 deletion syndrome (22q11.2DS) (MIM \#192430/188400) is the most common microdeletion syndrome in humans, with an estimated prevalence of at least 1 in 4,000 live births. ${ }^{1,2}$ Penetrance of the associated hemizygous $22 \mathrm{q} 11.2$ deletion for any major feature is high, but expression is highly variable. ${ }^{2}$ Commonly associated abnormalities include congenital heart disease (CHD) and intellectual disability (ID). ${ }^{1-5}$ Psychiatric illnesses are the prominent later-onset conditions in 22q11.2DS; schizophrenia and anxiety disorders are particularly prevalent in adults. ${ }^{4,6}$ Individually, each of these associated conditions can affect functioning of individuals in the general population. ${ }^{7-10}$ Although most children with 22q11.2DS now live to adulthood, ${ }^{11}$ there are limited data on adult functioning in 22q11.2DS, including the phenotypic traits that are most strongly predictive of functional outcome. Such knowledge is needed to inform expectations of families and clinicians and to facilitate the design of targeted interventions to optimize adaptive functioning in adulthood for patients with this common genomic disorder.
In this study, we assessed the functional abilities of 100 adults with 22q11.2DS using the Vineland Adaptive Behavior Scales (VABS) and additional demographic proxies. We hypothesized the existence of widespread functional deficits that would have an impact on all major aspects of adult life. Secondary hypotheses of clinical interest, given the high interindividual variability of $22 \mathrm{q} 11.2 \mathrm{DS},{ }^{2}$ were that ID, serious $\mathrm{CHD}$, and psychiatric illnesses would be independent predictors of impaired functioning.

\section{MATERIALS AND METHODS}

\section{Subjects and phenotypic assessments}

A total of 100 Canadian adults (aged 17 years and older) with 22q11.2DS ( $n=46$ male, 46.0\%) were included in this study. The mean age of patients at time of adaptive functioning assessment was 28.8 years ( $\mathrm{SD}=9.7$ years). All subjects met clinical screening criteria for 22q11.2DS, and 22q11.2 deletions were confirmed using standard methods. ${ }^{4,12}$ The mean age of molecularly confirmed 22q11.2DS diagnosis was 23.0 years $(\mathrm{SD}=$ 11.2 years). Nine subjects had 22q11.2 deletions that were likely

\footnotetext{
${ }^{1}$ Clinical Genetics Research Program, Centre for Addiction and Mental Health, Toronto, Ontario, Canada; ${ }^{2}$ Institute of Medical Science, University of Toronto, Toronto, Ontario, Canada; ${ }^{3}$ Department of Psychiatry, University of Toronto, Toronto, Ontario, Canada; ${ }^{4}$ Department of Psychiatry and Division of Cardiology, University Health Network, Toronto, Ontario, Canada; ${ }^{5}$ Department of Medicine, University Health Network, Toronto, Ontario, Canada. Correspondence: Anne S. Bassett (anne.bassett@utoronto.ca) 
transmitted from parents ( $n=6$ confirmed, $n=3$ probable by clinical diagnosis); the remaining 91 were known or presumed de novo mutations, depending on the availability of parents for molecular testing. The majority of subjects were ascertained through genetic, adult congenital cardiac, and psychiatric services using active screening and/or clinical referrals. ${ }^{13,14}$ Detailed phenotyping is described elsewhere. , $^{42,15,16}$ In brief, lifetime psychiatric diagnoses were determined by research psychiatrists (A.S.B., E.W.C.C.) according to the Diagnostic and Statistical Manual of Mental Disorders, fourth edition (DSM-IV) using direct semi-structured interview, collateral information from family members, and medical records as previously described. ${ }^{12}$ Subjects with schizophrenia or schizoaffective disorder $(n=$ $42)$ and psychotic disorder not otherwise specified $(n=3)$ met the criteria for major psychotic disorders, collectively termed "schizophrenia" for this study. The mean age at onset of schizophrenia was 21.3 years ( $\mathrm{SD}=5.6$ years). Nearly one-third of the sample $(n=30,30.0 \%)$ had a lifetime history of a nonpsychotic mood and/or anxiety disorder. Eight of these cases were comorbid with a diagnosis of schizophrenia. None had a diagnosis of autism. One subject had a parent with schizophrenia. Subjects were classified using DSM-IV criteria as having average intellect $(n=9,9.0 \%)$, or borderline $(n=48,48.0 \%)$, or mild $(n=$ $43,43.0 \%)$ ID. ${ }^{5}$ Individuals with moderate to severe ID were not included in this study in order to minimize possible floor effects on the VABS; this level of intellect is uncommon in 22q11.2DS. ${ }^{5}$ We classified CHD as before and considered only serious CHD (e.g., tetralogy of Fallot)..$^{17}$ Of the 39 subjects (39.0\%) with serious CHD, all but two had previously had corrective cardiac surgery. Most subjects had a degree of hypernasality ${ }^{2}$ and the majority had received speech therapy $(n=70,70.0 \%)$. Of the 46 subjects with velopharyngeal insufficiency and/or submucous cleft palate, 32 had their defects repaired surgically. Another four had repaired overt cleft palate. Informed consent was obtained in writing, and the study was approved by local research ethics boards.

\section{Neurocognitive and functional assessments}

Full-scale intelligence quotient (IQ) was assessed using the Wechsler Adult Intelligence Scale-Revised (WAIS-R; $n=48$, $48.0 \%)^{18}$ or the Wechsler Adult Intelligence Scale III (WAIS III; $n=51,51.0 \%) .{ }^{19}$ Historical IQ was used for one patient unavailable for testing. All psychometric tests were administered by a trained psychometrist. ${ }^{5}$ To investigate adaptive functioning, an individual who was a direct caregiver, spouse, or otherwise well acquainted with the adult with 22q11.2DS was interviewed using the VABS: Interview Edition, Expanded Form..$^{20}$ We previously reported VABS results for 58 individuals. ${ }^{12}$ The VABS was initially created for use in pediatric populations, but has since been used and validated in many studies of adults with ID and/or genetic syndromes. ${ }^{12,20-24}$ The VABS includes three main domains: Daily Living Skills (practical skills necessary for selfcare, domestic tasks, and community functioning), Socialization (skills needed to get along with others, engage in leisure activities, and regulate emotions and behavior), and Communication (skills required for receptive, expressive, and written language). The Adaptive Behavior Composite (ABC) score, a measure of global adaptive functioning of the individual, is calculated from these three domains. The VABS provides standard scores (mean $=100, \mathrm{SD}=15)$. Higher scores indicate better functioning. The proportion of participants with abnormal scores on the VABS was determined using a 1.5-SD cutoff, with scores below 78 indicating functional difficulty. ${ }^{25}$ Demographic data on crosssectional functioning were also collected through interviews with the caregiver and/or patient. We used the most current demographic data available for each subject in this study; the data were collected within 2 years of VABS assessment for 91 subjects.

\section{Statistical analyses}

Multivariate linear regression models for $\mathrm{ABC}$ and all three domains were used to identify phenotypic predictors of adult functioning in 22q11.2DS. Based on the major phenotypes of 22q11.2DS known to affect functioning in other general populations, ${ }^{7-10}$ the selected clinical covariates considered in addition to age and sex were overall full-scale IQ, and diagnosis of CHD, schizophrenia, and nonpsychotic mood and anxiety disorders. Post hoc $t$-tests were used to assess the individual regression coefficients. Children with 22q11.2DS have been reported to have higher verbal IQ scores than performance IQ scores, ${ }^{26,27}$ therefore we also tested verbal and performance IQ as predictors of functioning in place of overall IQ in our regression model.

In addition to overall descriptive statistics, we compared the adaptive functioning skills of subjects with average to borderline intellect $(n=57)$ with those with mild ID $(n=43)$. To assess functional outcomes and demographic and clinical variables, we used $\chi^{2}$ tests for categorical variables and independent or paired $t$-tests for continuous variables. Pearson's correlation was used to assess the strength of the relationship between overall IQ and ABC score. To assess functioning in subjects with the mildest neuropsychiatric expression, we repeated the analyses after restricting to subjects with average to borderline intellect who did not have schizophrenia $(n=38)$. All statistical analyses were two-tailed and performed with SAS version 9.3 software (SAS Institute, Cary, NC), with statistical significance defined as $P<0.05$.

\section{RESULTS}

The mean total IQ of the 100 adults with 22q11.2DS studied $(71.7, \mathrm{SD}=9.1)$ was in the lower range of borderline ID. There was no significant difference between mean verbal IQ $(73.7, \mathrm{SD}$ $=9.4)$ and mean performance IQ $(72.6, \mathrm{SD}=8.8 ; t=1.6, \mathrm{df}$ $=95, P=0.1120)$. Those with average to borderline intellect (mean overall $\mathrm{IQ}=77.3, \mathrm{SD}=7.7$ ) did not significantly differ in age ( $t=0.8, \mathrm{df}=98, P=0.4114)$, sex $\left(\chi^{2}=0.1, \mathrm{df}=1, P=\right.$ $0.7704)$, lifetime history of nonpsychotic mood and/or anxiety disorders $\left(\chi^{2}=0.1, \mathrm{df}=1, P=0.7914\right)$, or presence of $\mathrm{CHD}\left(\chi^{2}\right.$ $=3.1, \mathrm{df}=1, P=0.0770)$ from those with mild ID (mean overall $\mathrm{IQ}=64.3, \mathrm{SD}=4.0$ ). The mild ID subgroup had a significantly 
greater proportion of subjects with schizophrenia $(60.5 \%$ vs. $\left.33.3 \% ; \chi^{2}=6.2, \mathrm{df}=1, P=0.0125\right)$. As expected, ${ }^{15}$ mean overall IQ was significantly lower in patients with schizophrenia than in those who were nonpsychotic $(68.6(\mathrm{SD}=8.0)$ and 74.3 (SD $=9.1$ ), respectively; $t=-3.3, \mathrm{df}=98, P=0.0013)$, as were verbal IQ (70.4 ( $\mathrm{SD}=98.5)$ and $76.2(\mathrm{SD}=9.2)$, respectively; $t=-3.1$, $\mathrm{df}=94, P=0.0024)$ and performance IQ $(69.0(\mathrm{SD}=6.4)$ and $75.4(\mathrm{SD}=9.3)$, respectively; $t=-3.8, \mathrm{df}=94, P=0.0003)$.

\section{Adaptive functioning in 22q11.2DS}

Consistent with our hypothesis, the VABS scores revealed widespread functional deficits in our adult cohort with 22q11.2DS. The mean scores for the Daily Living Skills, Socialization, and Communication domains were $81.3(\mathrm{SD}=19.2), 67.2(\mathrm{SD}=$ $16.9)$, and $60.5(S D=20.7)$, respectively. The mean $\mathrm{ABC}$ score was $65.2(\mathrm{SD}=17.3)$, and the majority of participants scored in the functional difficulty range on overall functioning $(n=76,76.0 \%)$.

A significant predictive model $(P<0.001)$ emerged for the VABS ABC score (adjusted $R^{2}=0.38$ ) and all three of its domains: Daily Living Skills (adjusted $R^{2}=0.32$ ), Socialization (adjusted $R^{2}=0.22$ ), and Communication (adjusted $R^{2}=0.35$ ). As hypothesized, IQ and schizophrenia were significant predictors of overall adult functioning (Table 1). However, contrary to our hypothesis, neither CHD nor a history of mood or anxiety disorder had a significant impact on adaptive functioning. IQ and schizophrenia were also predictive of Daily Living Skills and Socialization scores, whereas IQ alone was a significant predictor of Communication scores (Table 1). No other variable achieved significance as a predictor for any of the three domains (Table 1). The exploratory regression models including verbal or performance IQ in place of overall IQ showed similar results (data not shown). Also, an exploratory analysis comparing functioning of those with and without a palatal abnormality showed no significant differences between these subgroups (data not shown).

Secondary analyses of the VABS standard scores showed the predominant effects of lower intellect on functioning across all domains. The average to borderline intellect subgroup performed significantly better overall and in all three domains than those with mild ID, and significantly fewer showed functional difficulty (Table 2). Restricting the analyses to the 38 individuals in the average to borderline intellect group, who were nonpsychotic, showed only slightly higher mean VABS scores, and half $(n=19)$ exhibited overall functional difficulty (Table 2). Notably, Daily Living Skills appeared to be a relative strength regardless of neuropsychiatric subgroup (Table 2). No difference was found in the pattern of results when IQ was used to define the two subgroups (mild ID, IQ $\leq 70$; borderline to average intellect, IQ > 70; data not shown).

Given the large variability in VABS scores observed in the two neurocognitive subgroups (Table 2), we examined more closely the relationship between IQ and overall functioning. Although IQ was well correlated with the VABS ABC score (all patients, $r=0.60, P<0.0001$; schizophrenia patients, $r=0.60$, $P<0.0001$; nonpsychotic patients, $r=0.52, P<0.0001)$, there was considerable variability in individual scores (Figure 1). For example, a small cluster of five individuals exceeded functional expectations compared with what would be predicted by IQ (Figure 1). Four were female and all five lived in a stable home environment with relatives or a spouse. Vocationally, they were students, employed, or had previously worked full time. Four had CHD, and four had an effectively treated mood/anxiety disorder. None had schizophrenia. Of note, all but two of the 24 individuals who functioned above the functional difficulty range on the $\mathrm{ABC}$ score had no psychotic illness (Figure 1).

\section{Functional descriptive variables}

Tables 3 and 4 show the details of specific functioning in personal relationships, vocations, and financial support for our sample. In total, $18 \%(n=18)$ of 22q11.2DS patients were or had previously been married, the majority of whom $(n=14)$ had at least one child. ${ }^{28}$ More than one in five had a driver's license (Table 3). Most patients who were married, had children, or were licensed to drive had average to borderline intellect and no psychotic illness. More than half of all patients lived with their parents and/or another relative (mean age $=24.6, \mathrm{SD}=$ 6.7, range 17.3-44.3 years), irrespective of intellectual functioning (Table 3). Thirteen (22\%) of those who lived with family were age 30 or older. They varied in severity of neuropsychiatric

Table 1 Summary of multivariate regression analysis of Vineland Adaptive Behavior Scales scores of 100 adults with 22q11.2 deletion syndrome

\begin{tabular}{|c|c|c|c|c|c|c|c|c|}
\hline & & & \multicolumn{6}{|c|}{ Vineland Adaptive Behavior Scales domains } \\
\hline & \multicolumn{2}{|c|}{ Adaptive behavior composite } & \multicolumn{2}{|c|}{ Daily living skills } & \multicolumn{2}{|c|}{ Socialization } & \multicolumn{2}{|c|}{ Communication } \\
\hline & B & $P$ & $B$ & $P$ & $B$ & $P$ & $B$ & $P$ \\
\hline Schizophrenia & 8.51 & 0.0130 & 8.55 & 0.0299 & 10.38 & 0.0054 & 4.32 & 0.2931 \\
\hline Mood/anxiety disorders & -0.02 & 0.9963 & -2.37 & 0.5293 & -0.46 & 0.8963 & 0.04 & 0.9927 \\
\hline Sex & 0.56 & 0.8437 & -0.02 & 0.9949 & -0.84 & 0.7835 & 1.70 & 0.6208 \\
\hline
\end{tabular}

$B$, regression coefficient; CHD, congenital heart disease; IQ, intelligence quotient; $P, P$ value for regression coefficient post hoc $t$-tests.

IQ and age are continuous variables; all others are dichotomous categorical variables.

Bold values indicate statistical significance $(P<0.05)$. 


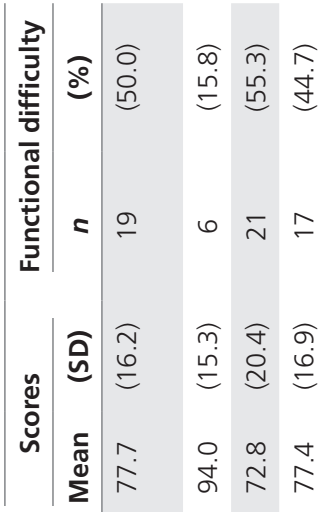

ฉ 용
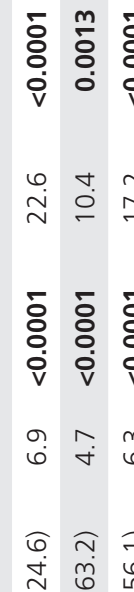

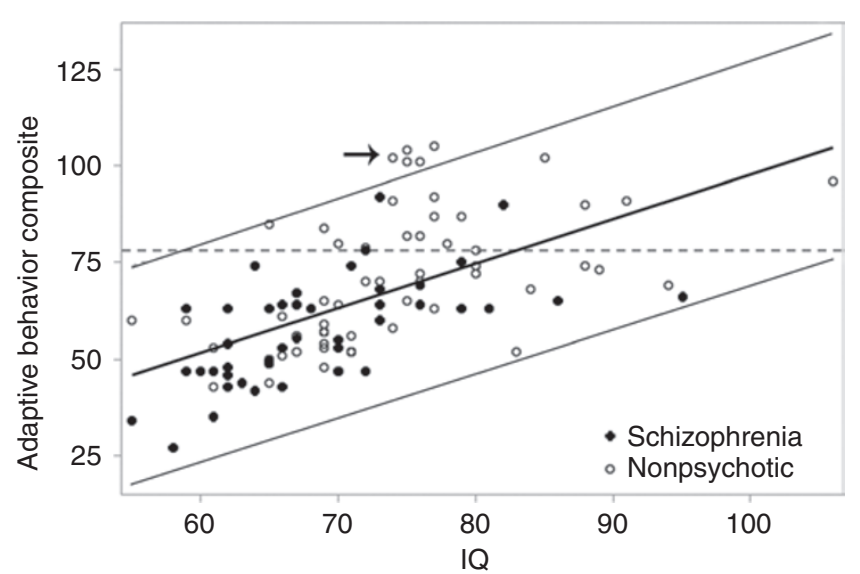

Figure 1 Full-scale IQ correlates with Vineland Adaptive Behavior Scales Adaptive Behavior Composite score $(r=0.60, n=100)$ in adults with 22q11.2 deletion syndrome. Regression and predicted interval lines $(95 \%)$ are shown. Subjects with an overall functioning score of less than 78 (dashed line) have functional deficits. Arrow denotes five subjects who have significantly higher functional scores than predicted by IQ. IQ, intelligence quotient.

phenotype; there were roughly equal numbers with mild ID and psychosis $(n=4)$ and average to borderline intellect, with $(n=$ 4 ) or without psychosis $(n=5)$.

Financial assistance from family and government sources was common (Table 4). Although more than two-thirds of the total sample had most often been employed during adulthood ( $n=66,66 \%)$, a minority was financially independent (Table 4). This was true even for individuals who had neither ID nor psychosis (Table 4). Parents and other relatives were the primary source of financial support for $16 \%(n=16)$ of the sample. Those who were married were typically supported at least in part by their spouse $(n=10 / 12,83.3 \%)$. The majority of the sample, however, was on fixed income $(n=61,61.0 \%)$. Fewer subjects with average to borderline intellect were on fixed income $\left(\chi^{2}=6.7, \mathrm{df}=1, P=0.0094\right)$ than those with mild ID (Table 4).

The types of jobs held by patients were diverse. Of note, few required previous experience or formal training. These included work in fast-food restaurants and coffee shops, general labor (e.g., worker in a loading bay), or maintenance positions (e.g., janitorial or cleaning staff). Less commonly, patients held jobs requiring more advanced skills, such as working with children (e.g., child care), with the general public (e.g., receptionist, store clerk), or in technical positions (e.g., computer store staff, call center worker, web designer). The sample also included a few trade workers (e.g., pipe fitter, wood worker, hair dresser). The highest professional designation attained was that of a school teacher.

\section{DISCUSSION}

Consistent with our primary hypothesis, 22q11.2DS in adulthood is characterized by widespread functional deficits that have an impact on most major aspects of daily life. A substantial proportion of the 100 adults with 22q11.2DS studied were 
Table 3 Social functioning of 100 adults with 22q11.2 deletion syndrome

\begin{tabular}{|c|c|c|c|c|c|c|}
\hline & \multicolumn{2}{|c|}{$\begin{array}{l}\text { Mild intellectual disability } \\
\qquad(n=43)\end{array}$} & \multicolumn{2}{|c|}{$\begin{array}{l}\text { Average to borderline intellect } \\
\qquad(n=57)\end{array}$} & \multicolumn{2}{|c|}{$\begin{array}{l}\text { average to borderline subgroup } \\
\qquad(n=38)\end{array}$} \\
\hline & $n$ & $(\%)$ & $n$ & (\%) & $n$ & $(\%)$ \\
\hline Married or common law & 2 & $(4.7)$ & 10 & $(17.5)$ & 9 & $(23.7)$ \\
\hline Separated, divorced, or annulled & 1 & (2.3) & 5 & (8.8) & 3 & (7.9) \\
\hline \multicolumn{7}{|l|}{ Home environment } \\
\hline House or apartment & 30 & $(69.8)$ & 51 & $(89.5)$ & 38 & $(100.0)$ \\
\hline Boarding house/group home & 11 & $(25.6)$ & 4 & (7.0) & 0 & - \\
\hline Hospital/treatment facility & 2 & $(4.7)$ & 2 & (3.5) & 0 & - \\
\hline Parent(s) and/or other relative(s) & 26 & $(60.5)$ & 32 & $(56.1)$ & 25 & $(65.8)$ \\
\hline Unrelated roommates & 13 & $(30.2)$ & 8 & $(14.0)$ & 0 & - \\
\hline Driver's license ownership & 1 & (2.3) & 21 & (36.8) & 19 & $(50.0)$ \\
\hline
\end{tabular}

Table 4 Employment and financial status of 100 adults with 22q11.2 deletion syndrome

\begin{tabular}{|c|c|c|c|c|c|c|}
\hline & \multicolumn{2}{|c|}{$\begin{array}{l}\text { Mild intellectual disability } \\
\qquad(n=43)\end{array}$} & \multicolumn{2}{|c|}{$\begin{array}{l}\text { Average to borderline intellect } \\
\qquad(n=57)\end{array}$} & \multicolumn{2}{|c|}{$\begin{array}{l}\text { Nonpsychotic subjects from the } \\
\text { average to borderline subgroup } \\
\qquad(n=38)\end{array}$} \\
\hline & $n$ & $\%$ & $n$ & $\%$ & $n$ & $\%$ \\
\hline Employed & 24 & $(55.8)$ & 42 & $(73.7)$ & 28 & $(73.7)$ \\
\hline Student \pm outside work & 5 & $(11.6)$ & 8 & $(14.0)$ & 7 & $(18.4)$ \\
\hline Disabled/unable to work ${ }^{\mathrm{a}}$ & 11 & $(25.6)$ & 1 & $(1.8)$ & 0 & - \\
\hline \multicolumn{7}{|l|}{ Primary source of financial support } \\
\hline Own job only & 3 & $(7.0)$ & 10 & $(17.5)$ & 10 & $(26.3)$ \\
\hline Own and spouse/partner income & 1 & (2.3) & 6 & $(10.5)$ & 5 & $(13.2)$ \\
\hline
\end{tabular}

ancludes intellectual, psychiatric, and/or physical impairments.

bIncludes pensions/retirement income, government social assistance, and disability pensions.

impaired in overall adaptive functioning as measured by the VABS and this corresponded with real-life deficits, reflected by the relatively low proportion of individuals who supported themselves financially, developed long-term romantic relationships, or lived on their own. Employability and skills necessary for activities of daily living appear to be areas of relative strength. There is however wide interindividual variability in functional abilities in adults with 22q11.2DS, which is mediated mainly by intellectual level and, to a lesser extent, by the presence or absence of schizophrenia.

Contrary to our hypotheses, other treatable psychiatric illnesses, such as mood and anxiety disorders, and CHD did not have a significant impact on functional abilities. This may be related to effective management and regular follow-up of these conditions. ${ }^{211}$ This also appeared to be the case for major palatal anomalies. Consistent with the findings of a recent study of 
reproductive fitness in $22 \mathrm{q} 11.2 \mathrm{DS},{ }^{28}$ our results suggest that the relative impact of serious CHD, such as tetralogy of Fallot, and its surgical repair on functioning in adulthood for patients with $22 \mathrm{q} 11.2 \mathrm{DS}$ is minimal. These results support a previous finding that neurodevelopmental deficits observed in children with $\mathrm{CHD}^{8}$ may be driven in large part by the effects of associated genetic syndromes such as 22q11.2DS. ${ }^{29}$

As expected, IQ was highly correlated with the VABS scores but was not the sole predictor of functioning. ${ }^{20}$ Our sample included a small group of adults who functioned at a higher level than would be predicted by their IQ, suggesting that, at least for some individuals, effective long-term management of chronic conditions and appropriate social and vocational support may promote the achievement of improved functional levels. Employment, for example, has been found to be associated with improved adaptive functioning skills in individuals with ID. ${ }^{30,31}$ Employment rates have been reported to be low in adults with 22q11.2DS, however. A previous study including occupational data on 17 adults with 22q11.2DS reported that about $35 \%$ (5 men, 1 woman) were employed. ${ }^{32}$ All others were homemakers. Notably, most in that sample were diagnosed as parents following the diagnosis of an affected child and had relatively mild manifestation of the syndrome and a high level of functioning. ${ }^{32}$ Results were similar in another report of seven adults with 22q11.2DS. ${ }^{33}$ Although we did not assess the crosssectional vocational status of our subjects, we found that most adults with 22q11.2DS had been employed in some capacity for the majority of their adulthood, regardless of intellectual level. Relatively few, however, were financially independent. Future study is needed to address the reasons underlying this discrepancy, but from our clinical experience, difficulties maintaining steady employment due to medical or psychiatric issues, the types of jobs in which they are employed (e.g., lower-wage, part-time, or temporary work) and poor money management skills may be contributing factors.

Daily Living Skills as measured by the VABS was an overall area of relative strength in adults with 22q11.2DS. This is consistent with previous reports using this assessment tool in other populations with various psychiatric illnesses and/or neurocognitive impairments. ${ }^{21-24}$ Of note, studies of children with 22q11.2DS have generally not shown this. ${ }^{34-36}$ The mean scores reported for children and adolescents in the literature on the Daily Living Skills domain were substantially (11-22 points) lower than the mean scores of adults in our sample, despite similar levels of intellect. ${ }^{34-36}$ Children with 22q11.2DS and a relatively high IQ, however, tend to score similarly well on the Daily Living Skills domain as the adults in our sample with average to borderline intellect. ${ }^{37}$ This suggests that there may be gains in this domain later in development and with maturity in 22q11.2DS, particularly in patients with lower intellect. Such changes in adaptive functioning skills could parallel the reported differences between the cognitive profiles of children and adults with 22q11.2DS. Performance IQ has been reported to be lower than verbal IQ in children, ${ }^{26,27}$ but not in adolescents or adults with 22q11.2DS. ${ }^{27,38}$ Alternatively, the higher Daily
Living Skills scores that we found in adults may be a function of differences in the sampled 22q11.2DS populations. For example, psychiatric conditions in our adults receiving psychiatric treatment may have had a modifying effect on functioning in this domain as compared with children and adolescents where there may be emerging and untreated psychiatric symptoms. ${ }^{35}$

There may be other age-related changes in adaptive functioning in 22q11.2DS. Although one study reported that girls tend to have a higher level of cognitive functioning and performed better on the VABS than boys, ${ }^{34}$ we found no effect of sex on functional abilities. Our results therefore suggest that any functional advantage that females may have in childhood is not maintained into adulthood. There have been no systematic reports on functioning in older or elderly adults with $22 \mathrm{q} 11.2 \mathrm{DS}$ to date, but there is some evidence from a case series of six patients with 22q11.2DS that cognitive abilities and adaptive skills decline with age, at least in some patients with treatment-resistant psychosis. ${ }^{39}$ Large scale, longitudinal studies are needed to investigate changes in functioning in individuals with 22q11.2DS over the lifespan.

\section{Study advantages and limitations}

With improved pediatric survival in 22q11.2DS, ${ }^{11}$ interests are shifting to understanding the long-term outcomes of this patient group. This is the first systematic study of adaptive functioning in a large cross-sectional sample of adults with 22q11.2DS and the first to test phenotypic predictors of functioning. Enrichment for schizophrenia, serious CHD, mood/anxiety disorders, and ID in this sample enhanced power for examining the effects of each of these variables on adaptive functioning. The prevalence of serious CHD was lower in our cohort than in others, where ascertainment primarily occurs through cardiac clinics and includes all forms of CHD. Our ascertainment strategies meant that we avoided oversampling transmitting parents. No study, however, can ensure a straightforward generalization of its overall descriptive statistics on functioning to the total population of individuals with 22q11.2 deletions because the true prevalences of major features remain unknown.

There were limitations of our study. Our study design focused on major phenotypes of 22q11.2DS with prior evidence in other general populations of having an impact on functioning. The selected variables explained $\sim 22-38 \%$ of the variance in the VABS domain and composite scores. Other variables that could contribute to adaptive functioning abilities in adults with 22q11.2DS remain to be identified. We did not examine the effect of psychiatric diseases other than schizophrenia and mood/anxiety disorders in 22q11.2DS. However, these other conditions are less common in our sample. ${ }^{6}$ Small numbers of subjects with transmitted 22q11.2 deletions $(n=9)$ or a parent with schizophrenia $(n=1)$ precluded the possibility of assessing the potential impact of these factors on long-term functioning. Future studies may address the impact of other potentially important variables on adult functioning such as subtle brain malformations and other neuropsychiatric phenomenology. ${ }^{2}$ The influence of early interventions on long-term outcomes in 
22q11.2DS also warrants systematic investigation but was outside the scope of this study.

We found no effect of age on functioning in our sample. Longitudinal studies are needed to assess adaptive functioning across the lifespan of individuals with 22q11.2DS as our results and those in studies of children together suggest that the level of functioning and predictors of functioning could change over time. Many of our subjects were not formally diagnosed until adulthood and so future cohorts of adults with 22q11.2DS may have improved functional outcomes over those reported here due to earlier diagnosis and, correspondingly, earlier access to optimal medical interventions and educational supports. ${ }^{2}$ Exploratory analyses indicated that serious palatal anomalies did not appear to affect long-term functioning, but the important issue of optimizing communication skills requires further, ideally longitudinal, study. As with all interview-based assessments, the abilities of patients may have been over- or underestimated in individual cases; however, this would be unlikely to systematically affect the pattern of overall findings. A small number of nonpsychotic patients in this study may develop schizophrenia in the future. The effect of lifetime psychotic illness on functioning may thus have been slightly underestimated, however most adults in our sample were past the mean age of onset of psychosis.

\section{Implications}

These findings may help patients, their families, and health-care providers in developing reasonable expectations and long-term goals, and inform interventions to improve functioning and increase level of independence. The results highlight the need for increased support and services for adults with 22q11.2DS that could build on relative strengths in daily living skills and employment and that remediate or help compensate for relative weaknesses in social and communication abilities. The use of modern tools, for example, could be considered. Receptive and expressive communication may be more effective using indirect methods (e.g., a computer, recorder, a smartphone, written text, or pictures) as compared with direct, verbal communication. Patients with mild ID are likely to face greater challenges in overcoming functional difficulties than those with higher intellect. As for others with ID, the development of cognitive rehabilitation strategies may help to remediate cognitive deficits and facilitate skill development. ${ }^{30,40}$ In addition, optimized psychiatric care, especially for psychotic illnesses (e.g., help with adherence to medications), will likely be of benefit. ${ }^{2}$ Increasing social, communication, and vocational skill sets, and confidence in abilities may translate into improved employability and greater independence.

The relative strengths demonstrated by both the VABS and descriptive statistics suggest that many patients with 22q11.2DS may be most successful in occupations and recreational activities that use skills related to activities of daily living. These could include possibilities such as gardening, food preparation, cleaning, or other domestic chores. Encouraging and assisting patients with 22q11.2DS with job placements in areas in which they are most likely to succeed may help to increase employment rates, independence, and personal satisfaction.
Individuals with 22q11.2DS tend to have executive functioning skill deficits, ${ }^{5}$ but relatively strong rote memory, ${ }^{26,27}$ and so may be well-suited to structured or repetitive tasks. Hands-on training, demonstrations, and written instructions may also help patients succeed in the workforce, as we find that many adults with 22q11.2DS are better experiential than passive, auditory learners. Students with 22q11.2DS who struggle academically could consider programs with a co-op component, to encourage academic success and foster skill development. Proximity to home and access to facilities with public transportation should be considered for both vocational and recreational activities, given that most patients with 22q11.2DS do not have a driver's license. Given the high interindividuality in functioning in adults with 22q11.2DS, vocational and social support should be tailored according to individual needs and interests.

\section{CONCLUSION}

In summary, intellectual level and a diagnosis of schizophrenia are good but imperfect predictors of adult functioning, and the presence of nonpsychotic mood disorders or serious CHD does not significantly affect outcome in 22q11.2DS. The characteristic wide interindividual variability of clinical features associated with 22q11.2DS ${ }^{2}$ extends to functional abilities. Future studies are needed to assess functioning across the lifespan and determine other remediable factors that predict better functioning. Nevertheless, the results suggest some strategies that may help the ever-increasing cohorts of patients with diagnosed 22q11.2DS entering adulthood, and the families and clinicians who help care for them.

\section{ACKNOWLEDGMENTS}

This study was supported by Canadian Institutes of Health Research grants (MOP-79518, MOP-89066, and MOP-97800), a Frederick Banting and Charles Best Canada Graduate Scholarship (to N.J.B.), and a W. Garfield Weston Foundation grant (to A.S.B.). A.S.B. holds the Canada Research Chair in Schizophrenia Genetics and Genomic Disorders. The authors thank the patients and their families for their participation, Don Young for assistance with VABS administration, and Fiona Fu, Monica Torsan, and Gladys Wong for their assistance with data collection.

\section{DISCLOSURE}

The authors declare no conflict of interest.

\section{REFERENCES}

1. Goodship J, Cross I, LiLing J, Wren C. A population study of chromosome 22q11 deletions in infancy. Arch Dis Child 1998;79:348-351.

2. Bassett AS, McDonald-McGinn DM, Devriendt K, et al.; International 22q11.2 Deletion Syndrome Consortium. Practical guidelines for managing patients with 22q11.2 deletion syndrome. J Pediatr 2011;159:332-9.e1.

3. Swillen A, Devriendt K, Legius $E$, et al. Intelligence and psychosocial adjustment in velocardiofacial syndrome: a study of 37 children and adolescents with VCFS. J Med Genet 1997;34:453-458.

4. Bassett AS, Chow EW, Husted J, et al. Clinical features of 78 adults with 22q11 Deletion Syndrome. Am J Med Genet A 2005;138:307-313.

5. Chow EW, Watson M, Young DA, Bassett AS. Neurocognitive profile in 22q11 deletion syndrome and schizophrenia. Schizophr Res 2006;87: 270-278. 
6. Fung WL, McEvilly R, Fong J, Silversides C, Chow E, Bassett A. Elevated prevalence of generalized anxiety disorder in adults with 22q11.2 deletion syndrome. Am J Psychiatry 2010;167:998.

7. Parker G, Rosen A, Trauer T, Hadzi-Pavlovic D. Disability associated with mood states and comparator conditions: application of the Life Skills Profile measure of disability. Bipolar Disord 2007;9:11-15.

8. Massaro AN, El-Dib M, Glass P, Aly H. Factors associated with adverse neurodevelopmental outcomes in infants with congenital heart disease. Brain Dev 2008;30:437-446.

9. Salvador-Carulla L, Saxena S. Intellectual disability: between disability and clinical nosology. Lancet 2009;374:1798-1799.

10. Leifker FR, Bowie CR, Harvey PD. Determinants of everyday outcomes in schizophrenia: the influences of cognitive impairment, functional capacity, and symptoms. Schizophr Res 2009;115:82-87.

11. McDonald-McGinn DM, Sullivan KE. Chromosome 22q11.2 deletion syndrome (DiGeorge syndrome/velocardiofacial syndrome). Medicine (Baltimore) 2011;90:1-18.

12. Bassett AS, Caluseriu O, Weksberg R, Young DA, Chow EW. CatecholO-methyl transferase and expression of schizophrenia in 73 adults with 22q11 deletion syndrome. Biol Psychiatry 2007;61:1135-1140.

13. Fung WL, Chow EW, Webb GD, Gatzoulis MA, Bassett AS. Extracardiac features predicting 22q11.2 deletion syndrome in adult congenital heart disease. Int J Cardio/ 2008;131:51-58.

14. Bassett AS, Costain G, Fung WL, et al. Clinically detectable copy number variations in a Canadian catchment population of schizophrenia. J Psychiatr Res 2010;44:1005-1009.

15. Bassett AS, Marshall CR, Lionel AC, Chow EW, Scherer SW. Copy number variations and risk for schizophrenia in 22q11.2 deletion syndrome. Hum Mol Genet 2008;17:4045-4053.

16. Bassett AS, Chow EW, Husted J, et al. Premature death in adults with 22q11.2 deletion syndrome. J Med Genet 2009;46:324-330.

17. Billett J, Cowie MR, Gatzoulis MA, Vonder Muhll IF, Majeed A. Comorbidity, healthcare utilisation and process of care measures in patients with congenital heart disease in the UK: crosssectional, population-based study with case-control analysis. Heart 2008;94:1194-1199.

18. Wechsler D. Wechsler Adult Intelligence Scale-Revised. The Psychological Corporation: San Antonio, TX, 1981.

19. Wechsler D. Wechsler Adult Intelligence Scale-III (WAIS-III). The Psychological Corporation: San Antonio, TX, 1997.

20. Sparrow S, Balla D, Cicchetti D. Vineland Adaptive Behavior Scales. American Guidance Service: Circle Pines, 1984.

21. Carter AS, Volkmar FR, Sparrow SS, et al. The Vineland Adaptive Behavior Scales: supplementary norms for individuals with autism. J Autism Dev Disord 1998:28:287-302.

22. Di Nuovo SF, Buono S. Psychiatric syndromes comorbid with mental retardation: differences in cognitive and adaptive skills. J Psychiatr Res 2007:41:795-800.

23. Visootsak J, Rosner B, Dykens E, Tartaglia N, Graham JM Jr. Behavioral phenotype of sex chromosome aneuploidies: 48,XXYY, 48, XXXY, and 49,XXXXY. Am J Med Genet A 2007;143A:1198-1203.
24. Adams $D$, Oliver $C$. The relationship between acquired impairments of executive function and behaviour change in adults with Down syndrome. J Intellect Disabil Res 2010;54:393-405.

25. Limperopoulos C, Majnemer A, Shevell MI, et al. Functional limitations in young children with congenital heart defects after cardiac surgery. Pediatrics 2001;108:1325-1331

26. Jacobson C, Shearer J, Habel A, Kane F, Tsakanikos E, Kravariti E. Core neuropsychological characteristics of children and adolescents with 22q11.2 deletion. J Intellect Disabil Res 2010;54:701-713.

27. Philip N, Bassett A. Cognitive, behavioural and psychiatric phenotype in 22q11.2 deletion syndrome. Behav Genet 2011;41:403-412.

28. Costain G, Chow EW, Silversides CK, Bassett AS. Sex differences in reproductive fitness contribute to preferential maternal transmission of 22q11.2 deletions. J Med Genet 2011;48:819-824.

29. Zeltser I, Jarvik GP, Bernbaum J, et al. Genetic factors are important determinants of neurodevelopmental outcome after repair of tetralogy of Fallot. J Thorac Cardiovasc Surg 2008;135:91-97.

30. Su CY, Chen CC, Wuang YP, Lin YH, Wu YY. Neuropsychological predictors of everyday functioning in adults with intellectual disabilities. $J$ Intellect Disabil Res 2008;52(Pt 1):18-28.

31. Stephens DL, Collins MD, Dodder RA. A longitudinal study of employment and skill acquisition among individuals with developmental disabilities. Res Dev Disabil 2005;26:469-486.

32. McDonald-McGinn DM, Tonnesen MK, Laufer-Cahana A, et al. Phenotype of the 22q11.2 deletion in individuals identified through an affected relative: cast a wide FISHing net! Genet Med 2001;3:23-29.

33. Lima K, Følling I, Eiklid KL, Natvig S, Abrahamsen TG. Age-dependent clinical problems in a Norwegian national survey of patients with the 22q11.2 deletion syndrome. Eur J Pediatr 2010;169:983-989.

34. Antshel KM, AbdulSabur N, Roizen N, Fremont W, Kates WR. Sex differences in cognitive functioning in velocardiofacial syndrome (VCFS) Dev Neuropsychol 2005;28:849-869.

35. Debbané M, Glaser B, David MK, Feinstein C, Eliez S. Psychotic symptoms in children and adolescents with 22q11.2 deletion syndrome: Neuropsychological and behavioral implications. Schizophr Res 2006;84:187-193

36. Gothelf D, Feinstein C, Thompson T, et al. Risk factors for the emergence of psychotic disorders in adolescents with 22q11.2 deletion syndrome. Am J Psychiatry 2007;164:663-669.

37. Roizen NJ, Higgins AM, Antshel KM, Fremont W, Shprintzen R, Kates WR. 22q11.2 deletion syndrome: are motor deficits more than expected for IQ level? J Pediatr 2010;157:658-661.

38. van Amelsvoort $\mathrm{T}$, Henry J, Morris R, et al. Cognitive deficits associated with schizophrenia in velo-cardio-facial syndrome. Schizophr Res 2004;70:223-232.

39. Evers LJ, De Die-Smulders CE, Smeets EE, Clerkx MG, Curfs LM. The velo-cardio-facial syndrome: the spectrum of psychiatric problems and cognitive deterioration at adult age. Genet Couns 2009;20:307-315.

40. Perrig WJ, Hollenstein M, Oelhafen S. Can we improve fluid intelligence with training on working memory in persons with intellectual disabilities? $J$ Cogn Edu Psychol 2009;8:148-164. 